\title{
Chronic treatment with vascular endothelial growth factor preserves agonist-evoked vascular responses in the streptozotocin-induced diabetic rat
}

Received: 12 August 2005 / Accepted: 25 November 2005 / Published online: 23 February 2006

C) Springer-Verlag 2006

\begin{abstract}
Aims/hypothesis: Vascular dysfunction is a hallmark of diabetes mellitus and endothelial dysfunction is considered to be a key early component of vascular dysfunction. Attenuated agonist-evoked responses are considered to be a barometer of endothelial/vascular dysfunction. We sought to determine whether vascular endothelial growth factor (VEGF) could prevent dysfunction from developing in the streptozotocin (STZ)-induced rat model of type 1 diabetes. Materials and methods: One week after induction of diabetes, STZ rats began a 4-week treatment protocol of twice-weekly i.v. injections of $2 \mu \mathrm{g}$ VEGF or inactivated VEGF. Corresponding non-diabetic rats served as controls. Agonist-evoked vascular responses were recorded 1 day after the last treatment in anaesthetised rats. Results: Acetylcholine $(0.1-12.5 \mu \mathrm{g} / \mathrm{kg})$ evoked increases in superior mesenteric arterial conductance and decreases in mean blood pressure, while methoxamine $(12.5-100 \mu \mathrm{g} / \mathrm{kg})$ and endothelin-1 $(100-1,200 \mathrm{pmol} / \mathrm{kg})$ evoked decreases in superior mesenteric arterial conductance and increases in mean blood pressure. These responses to all three agonists were attenuated in STZ rats, and chronic treatment with VEGF improved these responses dramatically. Both the reduction in plasma nitrate and nitrite and the elevation in aortic superoxide associated with STZ diabetes were normalised with VEGF treatment. VEGF also prevented the apparent paradoxical increased endothelial nitric oxide synthase expression seen in untreated STZ rats. Conclusions/interpretation: Chronic treatment with VEGF early in diabetes is able to prevent the attenuated agonistevoked vascular responses in STZ rats and normalise the oxidative environment associated with the disease.
\end{abstract}

\footnotetext{
S. Bardal · D. Misurski $\cdot$ X. Qiu ·

K. Desai · J. R. McNeill (\)

Pharmacology, College of Medicine,

University of Saskatchewan,

A120 Health Sciences Building, 107 Wiggins Road,

Saskatoon, SK S7N 5E5, Canada

e-mail: Robert.McNeill@usask.ca

Tel.: +1-306-9666300

Fax: +1-306-9666220
}

Keywords Agonist responses - Basic science - Diabetes mellitus - Endothelium - Microvascular disease $\cdot$ Nitric oxide - Oxidative stress $\cdot$ Rat $\cdot$ Streptozotocin $\cdot$ VEGF

Abbreviations eNOS: endothelial nitric oxide synthase . ET-1: endothelin-1 · iVEGF: inactivated vascular endothelial growth factor - MBP: mean blood pressure NO: nitric oxide - SD: Sprague-Dawley ·

SMA: superior mesenteric artery - STZ: streptozotocin · VEGF: vascular endothelial growth factor

\section{Introduction}

Although they have differing aetiologies, both type 1 (insulin-dependent) and type 2 (non-insulin-dependent) diabetes mellitus share the common pathophysiological feature of consistently elevated plasma glucose and a constellation of resulting vascular-related disorders, including retinopathy, nephropathy and atherosclerosis [1]. The precursor to the overt vasculopathies characteristic of diabetes is endothelial dysfunction [2]. In streptozotocin (STZ)-induced diabetic rats, this endothelial dysfunction is characterised by attenuated agonist-evoked responses to acetylcholine [3]. The impaired acetylcholine-evoked responses appear to be related, at least in part, to inactivation of nitric oxide (NO) by reactive oxygen species, notably the superoxide anion [4].

A major focus of current treatment of diabetes is on the maintenance of blood glucose within a fairly narrow range, reflecting the findings of the Diabetes Control and Complications Trial $[5,6]$. For many diabetic patients, such tight glycaemic control is not feasible, and even those who diligently regulate their blood glucose are still vulnerable to the many vasculopathies that characterise the disease. Treatments that preserve vascular function would therefore be of obvious therapeutic benefit. Vascular endothelial growth factor (VEGF) is a potential candidate as it has been reported to upregulate superoxide dismutase in isolated endothelial cells [7]. In view of the altered acetylcholine-evoked vascular responses typically seen in 
the diabetic state $[3,8]$, we sought to determine whether chronic treatment with VEGF early in the disease can preserve 'normal' acetylcholine-evoked responses in these rats, despite their uncontrolled diabetes. We postulated that improved responses to acetylcholine in diabetic rats treated with VEGF would be accompanied by normalisation of superoxide anion and NO levels, and of endothelial nitric oxide synthase (eNOS) expression. In addition to impaired acetylcholine-evoked responses in diabetic models, attenuated pressor responses to vasoconstrictor agonists, such as methoxamine and endothelin (ET)-1, have been reported $[9,10]$. Indeed, we have observed attenuated responses to ET-1 and methoxamine both in vivo and in vitro in the isolated perfused mesenteric vascular bed [11]. Thus, for comparison with vasodilator responses evoked by acetylcholine, we also recorded vasoconstrictor responses to ET-1 and methoxamine.

\section{Materials and methods}

Experimental protocol

All experiments were carried out in accordance with the guidelines set down by the University Council on Animal Care and Supply (UCACS) at the University of Saskatchewan. Diabetes was induced in 9-week-old SpragueDawley (SD) rats (300-350 g; Charles River, Montreal, QC, Canada) using a single i.p. injection of STZ (Sigma Aldrich, Oakville, ON, Canada) $(55 \mathrm{mg} / \mathrm{kg}$ in $0.1 \mathrm{~mol} / 1$ citrate buffer, $\mathrm{pH} 4.5)$. SD rats injected with buffer alone served as controls. One week after injection, the occurrence of polyuria, polydipsia, polyphagia and elevated blood glucose $(>20 \mathrm{mmol} / \mathrm{l})$ confirmed the diabetic state. Blood glucose was assayed by the glucose oxidase method (One Touch Basic, Vancouver, BC, Canada). Beginning at the first week after STZ, animals were randomised into several groups: STZ rats treated with either VEGF-165 $(n=10)$ or inactivated VEGF (iVEGF) $(n=11)$ and corresponding control SD rats $(n=8$ in the group treated with VEGF, $n=9$ in the group treated with iVEGF) for the in vivo agonist protocol. Animals in the treatment group received $2 \mu \mathrm{g}$ of VEGF (Genentech, South San Francisco, CA, USA) into the tail vein twice weekly for 4 weeks. Control groups were given heat-inactivated VEGF over the same period. The dosing regimen was based on similar studies by Meurice et al., carried out using basic fibroblast growth factor in rabbits with hypercholesterolaemia and balloon injury [12, 13]. One full day was given between the end of the treatment protocol and the beginning of the in vivo agonist protocol. A parallel group of animals in each treatment arm were not subjected to surgery or instrumentation. One day after the end of treatment, blood was taken from each of the animals in these groups ( $n=7$ in each) for measurement of plasma nitrate and nitrite. Aortas were removed for western blot analysis and measurement of superoxide anion levels.

\section{In vivo haemodynamic responses}

At the end of the 4-week treatment protocol, at the 5-week stage of diabetes, rats in the in vivo protocol had their blood glucose taken and were anaesthetised with thiopental $(120 \mathrm{mg} / \mathrm{kg}$, i.p.) for surgery and the agonist protocol. Radiotelemetry devices (DataSciences, St Paul, MN, USA) for measurement of blood pressure were implanted first. Briefly, a 2-cm incision was made in the ventral hindlimb, and the tip of the telemetry device was advanced approximately $4-5 \mathrm{~cm}$ into the femoral artery. A catheter was also advanced into the femoral vein to serve as an injection port. After a 5-cm incision had been made from the base of the sternum to the lower abdomen, a transit-time flow probe (Transonic, Ithaca, NY, USA) was implanted on the superior mesenteric artery (SMA). Once stabilised, animals were transferred to the recording room and placed on receivers for constant monitoring of blood pressure by radiotelemetry. The flow probes were connected to receivers, and both flow and telemetry readings were recorded using the DataQuest acquisition system (Data Sciences, Minneapolis, MN, USA). Body temperature was maintained at $37^{\circ} \mathrm{C}$. Supplementary doses of thiopental $(1 \mathrm{mg})$ were administered i.v. when needed. Once a stable baseline had been achieved, bolus injections of acetylcholine $(0.1-12.5 \mu \mathrm{g} / \mathrm{kg})$, methoxamine $(12.5-100 \mu \mathrm{g} / \mathrm{kg})$ and ET-1 (100-1,200 pmol/kg) were given i.v. Upon completion of the agonist protocol, blood samples were drawn before the animals were killed, and kidney and heart weights taken after death. Sections of aorta were removed from some animals at random for histological analysis.

\section{Measurement of plasma nitrate and nitrite}

Levels of plasma nitrate and nitrite were obtained using the Nitrate/Nitrite Colorimetric Assay Kit (Cayman Chemical, Ann Arbor, MI, USA). In the in vitro protocol, blood samples were collected into $10 \%$ sodium citrate immediately before the animals were killed. The tubes were centrifuged at $4^{\circ} \mathrm{C}$ and $1,000 \mathrm{~g}$ for $5 \mathrm{~min}$, and recovered plasma was stored at $-70^{\circ} \mathrm{C}$. Subsequently, the samples were thawed and transferred to Nanosep $10 \mathrm{~K}$ Omega centrifuge filter tubes (Pall, Ann Arbor, MI, USA), which employ $10-\mathrm{kDa}$ molecular mass cut-off filters. Tubes were centrifuged at $4^{\circ} \mathrm{C}$ and $15,000 \mathrm{~g}$ for $20 \mathrm{~min}$ to remove the haemoglobin. The filtrate was then transferred in $40 \mu \mathrm{l}$ aliquots to a 96-well plate; each sample was performed in duplicate. To each well, $10 \mu \mathrm{l}$ of enzyme cofactor mixture and $10 \mu \mathrm{l}$ of nitrate reductase mixture were added, and the plate was covered and incubated at room temperature for $3 \mathrm{~h}$. When the incubation was complete, $50 \mu \mathrm{l}$ each of $2 \%$ sulfanilamide in $5 \%$ phosphoric acid and $0.2 \%$ naphthylethylenediamine dihydrochloride were added to each well. After allowing $10 \mathrm{~min}$ for optimal colour development, we read the absorbance at $540 \mathrm{~nm}$ using Fluoroskan Ascent plate reader (ThermoLabsystems, Franklin, MA, USA). 
Detection of superoxide anion by lucigenin chemiluminescence

Superoxide generation was measured in sections of aorta removed from rats not undergoing in vivo haemodynamic analysis. After isolation, the aorta was cleaned and

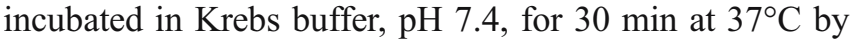
gassing with $95 \% \mathrm{O}_{2}-5 \% \mathrm{CO}_{2}$. The aorta was then transferred into a test-tube and incubated in darkness with $5 \mu \mathrm{mol} / 1$ lucigenin for $15 \mathrm{~min}$ at $37^{\circ} \mathrm{C}$. The chemiluminescence was measured every $30 \mathrm{~s}$ repeatedly over $5 \mathrm{~min}$ and an average value was calculated. After addition of $20 \mathrm{mmol} / \mathrm{l}$ Tiron (final concentration) (Sigma Chemical, Oakville, ON, Canada), a non-enzymatic scavenger of superoxide anion to quench all superoxide anion dependent chemiluminescence, the aorta was incubated at $37^{\circ} \mathrm{C}$ for $15 \mathrm{~min}$ and the repeated measurements were integrated over $5 \mathrm{~min}$. Superoxide anion generation was reported as $\mathrm{mLL} \min ^{-1} \mathrm{mg}^{-1}$ of aorta weight.

\section{Western blot analysis}

Western blot analyses were performed on sections of aorta removed from rats not undergoing in vivo haemodynamic analysis. Aortas were isolated and immediately stored in liquid nitrogen. The frozen tissue was then homogenised in lysis buffer containing $50 \mathrm{mmol} / \mathrm{l}$ Tris, $\mathrm{pH} 8,150 \mathrm{mmol} / \mathrm{l}$ $\mathrm{NaCl}, 0.1 \%$ SDS, $1 \%$ Triton $\mathrm{X}-100,1 \%$ sodium deoxycholate, and $1 \mathrm{mmol} / 1$ phenylmethylsulphonyl fluoride and centrifuged at $1,000 \mathrm{~g}$ at $4^{\circ} \mathrm{C}$ for $15 \mathrm{~min}$. The supernatant was transferred to a fresh tube and centrifuged at $30,000 \mathrm{~g}$ at $4^{\circ} \mathrm{C}$ for $30 \mathrm{~min}$. The protein concentration in the supernatant was determined using the Protein Assay Reagent (Bio-Rad Laboratories, Hercules, CA, USA), and $50 \mu \mathrm{g}$ of total protein was electrophoretically sizeseparated on a 7\% SDS-polyacrylamide gel and then transferred onto nitrocellulose membrane (Bio-Rad Laboratories) at $4{ }^{\circ} \mathrm{C}$ overnight. The membrane was blocked with 5\% non-fat dried milk in TBS-T (pH 7.6, $20 \mathrm{mmol} / 1$ Tris base, $137 \mathrm{mmol} / 1 \mathrm{NaCl}$, and $0.1 \%$ Tween 20) at room temperature for $1 \mathrm{~h}$ and then incubated with 1:2,000 diluted monoclonal mouse anti-eNOS antibody (Transduction Laboratories, Mississauga, ON, Canada) at $4^{\circ} \mathrm{C}$ overnight. The eNOS protein was detected by enhanced chemiluminescence (ECL; Amersham Pharmacia Biotech, Baie
d'Urfe, QC, Canada). The membrane was then stripped in stripping buffer containing $100 \mathrm{mmol} / \mathrm{l} 2$-mercaptoethanol, $2 \% \mathrm{SDS}, 62.5 \mathrm{mmol} / \mathrm{l}$ Tris- $\mathrm{HCl}, \mathrm{pH} 7.6$, at $50^{\circ} \mathrm{C}$ for $30 \mathrm{~min}$ and reprobed with 1:10,000 monoclonal mouse anti- $\beta$-actin antibody (Sigma Aldrich). The densitometric value of the samples was evaluated by densitometry (UNSCAN-IT; Silk Scientific Corporation, Orem, UT, USA).

\section{Statistical analysis}

Values are expressed as mean \pm SEM. Differences between results obtained with the agonist protocols were first determined by repeated measures ANOVA, which was followed by one-way ANOVA to test differences among multiple groups. In all cases, a $p$ value of less than 0.05 was considered significant.

\section{Results}

\section{Effects of VEGF on progression of diabetes}

Chronic treatment with VEGF or iVEGF had no effect on the progression of diabetes in STZ rats (Table 1). The animals displayed all the classic signs of diabetespolydipsia, polyphagia, polyuria (data not shown), minimal weight gain and elevated blood glucose (Table 1). Baseline mean blood pressure (MBP) was reduced and SMA conductance was elevated in 5-week STZ rats compared with their non-diabetic SD counterparts. Chronic treatment with VEGF preserved MBP at normal (non-diabetic) levels. In contrast, VEGF failed to maintain SMA conductance.

\section{Effects of VEGF on agonist-evoked responses in STZ rats}

The changes in SMA conductance and blood pressure in response to acetylcholine are shown in Fig. 1. In STZ diabetic rats, treatment with VEGF enhanced acetylcholine-evoked increases in SMA conductance and decreases in blood pressure compared with rats treated with iVEGF. In contrast, treatment of non-diabetic SD rats with VEGF failed to enhance acetylcholine-evoked responses. Interestingly, the response to the highest dose of acetylcholine

Table 1 Metabolic and baseline haemodynamic parameters in the VEGF study

\begin{tabular}{|c|c|c|c|c|c|c|}
\hline Group & Treatment & $\begin{array}{l}\text { Blood glucose } \\
(\mathrm{mmol} / \mathrm{l})\end{array}$ & Weight gain $(\mathrm{g})$ & Baseline MBP (mm Hg) & $\begin{array}{l}\text { Heart rate } \\
\text { (beats/min) }\end{array}$ & $\begin{array}{l}\text { SMA conductance }\left(\mathrm{ml} \mathrm{mm} \mathrm{Hg}^{-1}\right. \\
\left.\min ^{-1} \mathrm{~kg}^{-1}\right)\end{array}$ \\
\hline STZ & VEGF $n=10$ & $23.7 \pm 1.1^{\mathrm{a}}$ & $15.6 \pm 8.5^{\mathrm{a}}$ & $96.6 \pm 3.8^{\mathrm{b}}$ & $247.9 \pm 12.0^{\mathrm{a}}$ & $0.51 \pm 0.06^{\mathrm{a}}$ \\
\hline STZ & iVEGF $n=11$ & $23.2 \pm 1.2^{\mathrm{a}}$ & $14.6 \pm 6.7^{\mathrm{a}}$ & $86.2 \pm 2.6^{\mathrm{a}}$ & $227.3 \pm 10.1^{\mathrm{a}}$ & $0.53 \pm 0.07^{\mathrm{a}}$ \\
\hline $\mathrm{SD}$ & VEGF $n=8$ & $4.4 \pm 0.2$ & $149.8 \pm 8.2$ & $98.9 \pm 3.5$ & $298.9 \pm 15.4$ & $0.29 \pm 0.05$ \\
\hline $\mathrm{SD}$ & iVEGF $n=9$ & $4.3 \pm 0.2$ & $164.8 \pm 7.0$ & $99.2 \pm 5.2$ & $274.4 \pm 12.2$ & $0.26 \pm 0.02$ \\
\hline
\end{tabular}

Animals received $2 \mu \mathrm{g}$ VEGF i.v. (tail vein) twice weekly for 4 weeks, with inactivated VEGF (iVEGF) serving as a control. Values are mean \pm SEM

${ }^{\mathrm{a}} p<0.05$ vs corresponding SD group; ${ }^{\mathrm{b}} p<0.05$ vs corresponding iVEGF group 
was less in control iVEGF-treated STZ diabetic rats compared with iVEGF-treated non-diabetic SD rats $(p<0.05)$, but the responses to the highest dose of acetylcholine were similar in STZ and control SD rats after treatment with VEGF. Thus, VEGF treatment appeared to preserve acetylcholine-evoked responses in the STZ diabetic rats. In STZ diabetic rats, treatment with VEGF significantly enhanced the ET-1- (Fig. 2) and methoxamine- (Fig. 3) evoked decreases in SMA conductance and increases in blood pressure compared with iVEGF-treated rats. In control SD rats, ET-1- and methoxamine-evoked responses were either unchanged or enhanced only slightly by treatment with VEGF.

Effect of diabetes and VEGF treatment on plasma nitrate and nitrite levels

Plasma levels of nitrate and nitrite were much lower $(p<0.05)$ in STZ rats treated with iVEGF (control) compared with their non-diabetic SD counterparts (Fig. 4). Chronic treatment with VEGF significantly increased $(p<0.05)$ plasma levels of nitrate and nitrite in STZ rats compared with iVEGF-treated STZ rats (Fig. 4). In contrast, the nitrate and nitrite levels in VEGF-treated non-diabetic SD rats were actually lower than in nondiabetic SD rats treated with iVEGF.

Effects of diabetes and VEGF treatment on superoxide levels in thoracic aorta

Superoxide levels, as measured by chemiluminescence, were significantly elevated in the STZ diabetic group treated with iVEGF (control) compared with the corre- sponding non-diabetic SD group (Fig. 5). Chronic treatment with VEGF reduced the elevated superoxide levels in STZ rats to normal. Superoxide levels were unaffected by VEGF treatment in non-diabetic SD rats.

\section{Effects of diabetes and VEGF treatment on eNOS expression}

Increased expression of eNOS was evident in STZ diabetic rats treated with iVEGF control compared with their nondiabetic SD counterparts (Fig. 6). This enhanced eNOS expression was normalised by chronic treatment with VEGF. Interestingly, chronic treatment with VEGF actually enhanced eNOS expression in non-diabetic SD rats, in keeping with in vitro observations reported in previous studies $[14,15]$.

\section{Discussion}

The data in this article support the hypothesis that chronic treatment with VEGF at an early stage of STZ diabetes can preserve vascular function and that the preservation of vascular function is accompanied by changes in the oxidative environment, notably NO and superoxide anion levels. We found that agonist-evoked MBP and SMA responses to acetylcholine were attenuated in STZ rats, and that treatment with VEGF significantly improved these responses. Attenuated vascular responses to acetylcholine have been reported in numerous studies, in both clinical and experimental diabetes [3, 8]. Acetylcholine-evoked responses are used as a marker of endothelial function and, from a clinical perspective, represent a functional correlate of vascular damage. The results suggest that VEGF is able
Fig. 1 Percentage change in $\operatorname{MBP}(\mathbf{a}, \mathbf{b})$ and superior mesenteric artery conductance $(\mathbf{c}, \mathbf{d})$ in response to increasing doses of acetylcholine (ACh) administered i.v. in control (iVEGF, open triangles) or VEGF-treated (filled triangles) 5-week STZ (a, c) and agematched iVEGF- (open squares) and VEGF-treated (filled squares) non-diabetic SpragueDawley rats $(\mathbf{b}, \mathbf{d})$. Values are mean \pm SEM. VEGF-STZ vs iVEGF-STZ, $p<0.05$; VEGF-SD vs iVEGF-SD, $p=\mathrm{NS}$ for MBP and superior mesenteric artery conductance. Where error bars are missing they have been obscured by the data point a
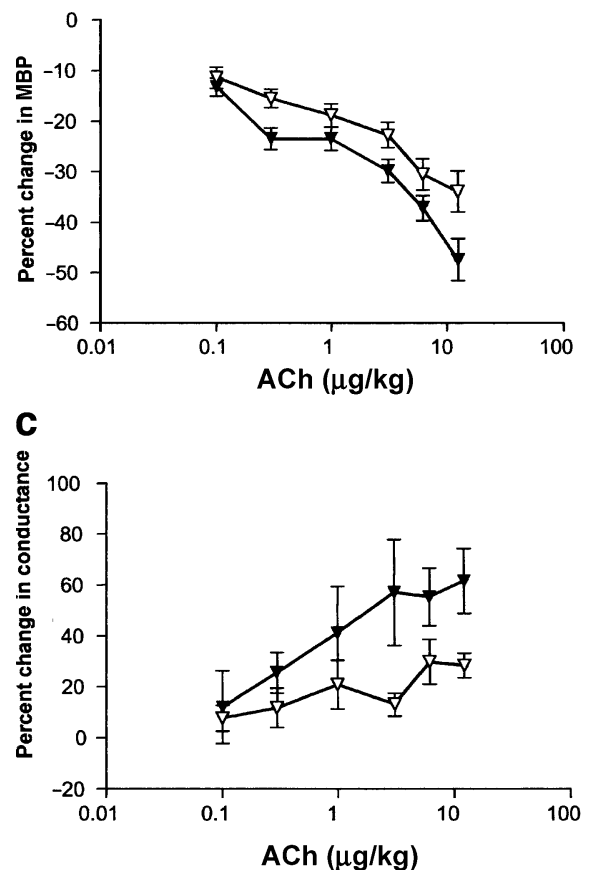

b
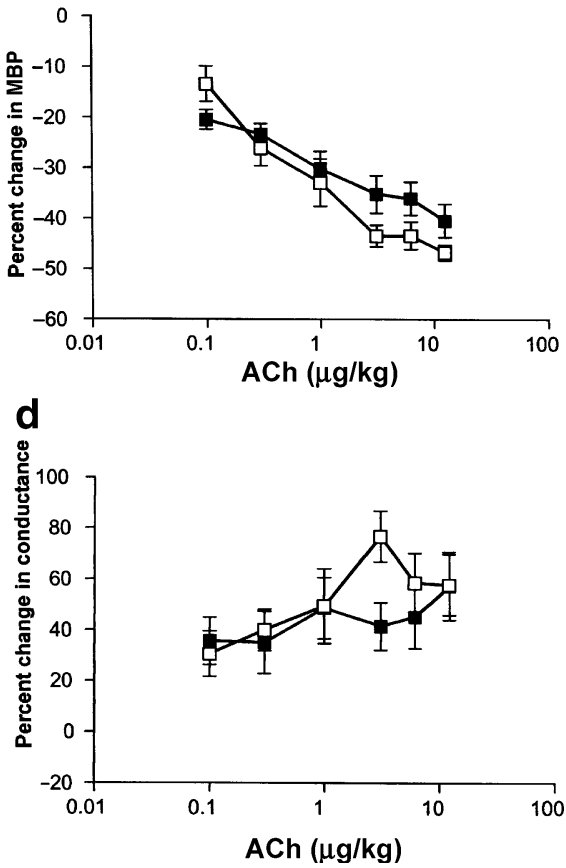
Fig. 2 Changes in $\operatorname{MBP}(\mathbf{a}, \mathbf{b})$ and percentage change in superior mesenteric artery conductance $(\mathbf{c}, \mathbf{d})$ in response to increasing doses of ET-1 administered i.v. in control (iVEGF, open triangles) or VEGF-treated (filled triangles) 5-week STZ $(\mathbf{a}, \mathbf{c})$ and in agematched iVEGF (open squares) and VEGF-treated (filled

squares) non-diabetic SpragueDawley rats $(\mathbf{b}, \mathbf{d})$. Values are mean \pm SEM. VEGF-STZ vs iVEGF-STZ, $p<0.05$ for MBP and SMAC; VEGF-SD vs iVEGF-SD, $p<0.05$ for MBP and $p=\mathrm{NS}$ for SMAC. Where error bars are not visible they have been obscured by the data point
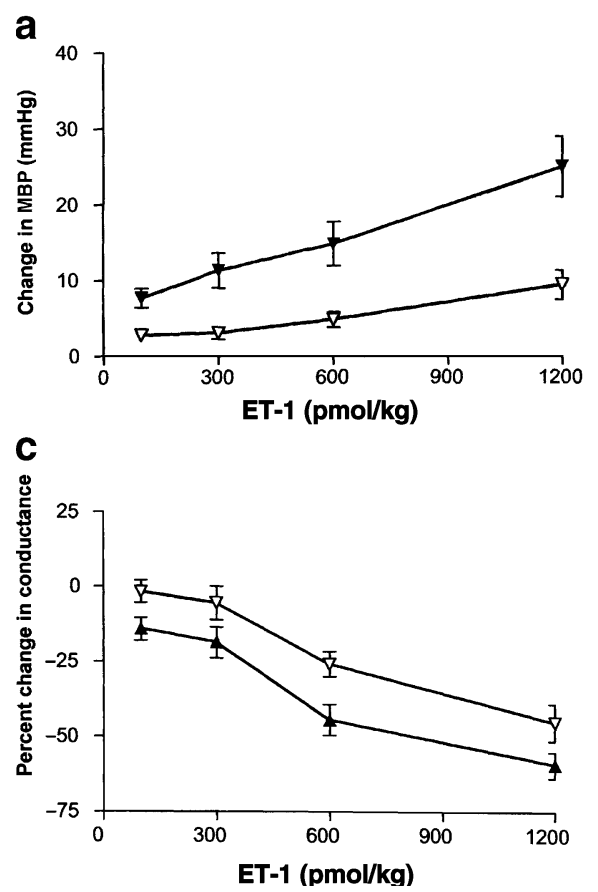

b

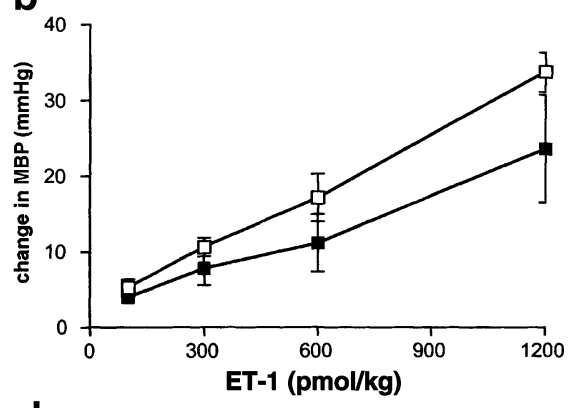

d

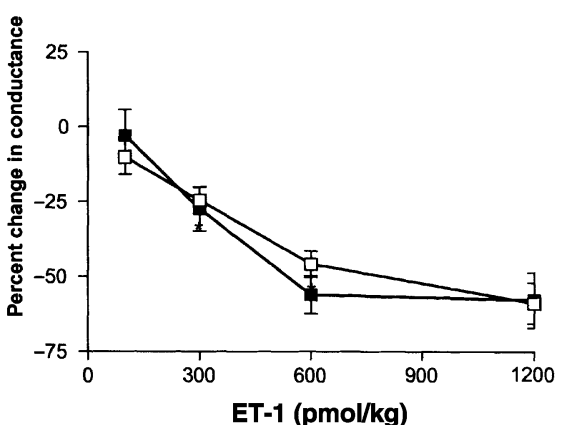

to preserve the functional integrity of the vasculature even when the hyperglycaemia associated with the diabetic state is uncontrolled.

Importantly, plasma nitrate and nitrite concentrations were lower in STZ diabetic rats than control SD rats, findings consistent with impaired NO function. Treatment with VEGF increased plasma nitrate and nitrite concentrations in STZ rats but not non-diabetic SD rats. Interestingly,
eNOS expression was upregulated as early as the 5 -week stage of STZ-induced diabetes, an observation that is in agreement with a previous study by Hink et al. [16]. The upregulation could be a compensatory response to support endothelial function. Chronic VEGF treatment reduced eNOS expression. Our findings of a reduction in eNOS expression accompanied by increased nitrate and nitrite levels and acetylcholine responsiveness may appear
Fig. 3 Changes in MBP (a, b) and superior mesenteric artery conductance $(\mathbf{c}, \mathbf{d})$ in response to increasing doses of methoxamine (MTX) administered i.v. in control (iVEGF, open triangles) or VEGF-treated (filled triangles) 5-week STZ $(\mathbf{a}, \mathbf{c})$ and in agematched iVEGF- (open squares) or VEGF-treated (filled squares) non-diabetic Sprague-Dawley rats $(\mathbf{b}, \mathbf{d})$. Values are mean \pm SEM. VEGF-STZ vs iVEGF$\mathrm{STZ}, p<0.005$ for MBP, $p<0.05$ for SMAC; VEGF-SD vS iVEGF-SD, $p=\mathrm{NS}$ for MBP and $p<0.05$ for SMAC. Where error bars are not visible they have been obscured by the data point a
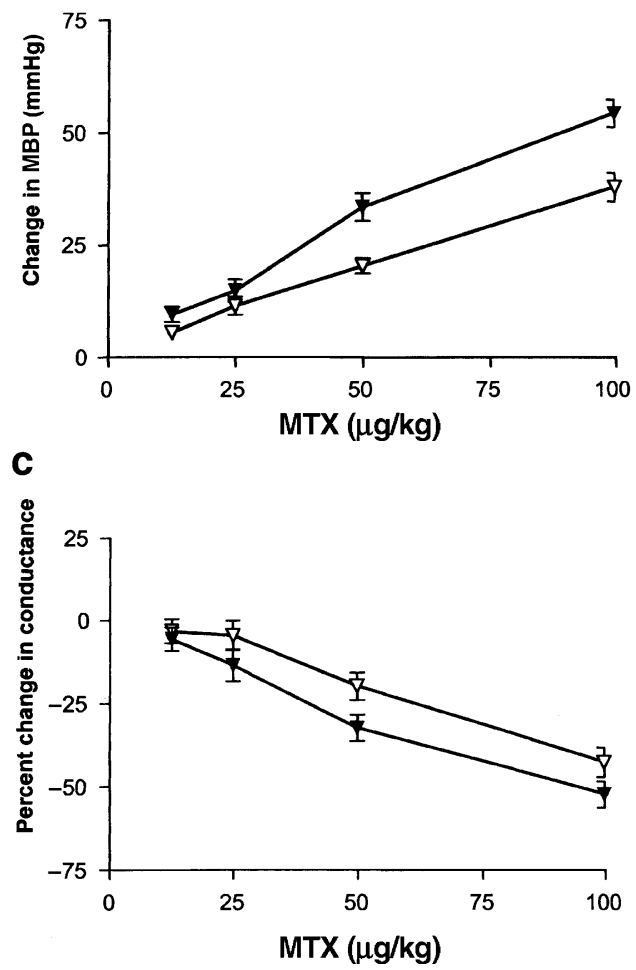

b
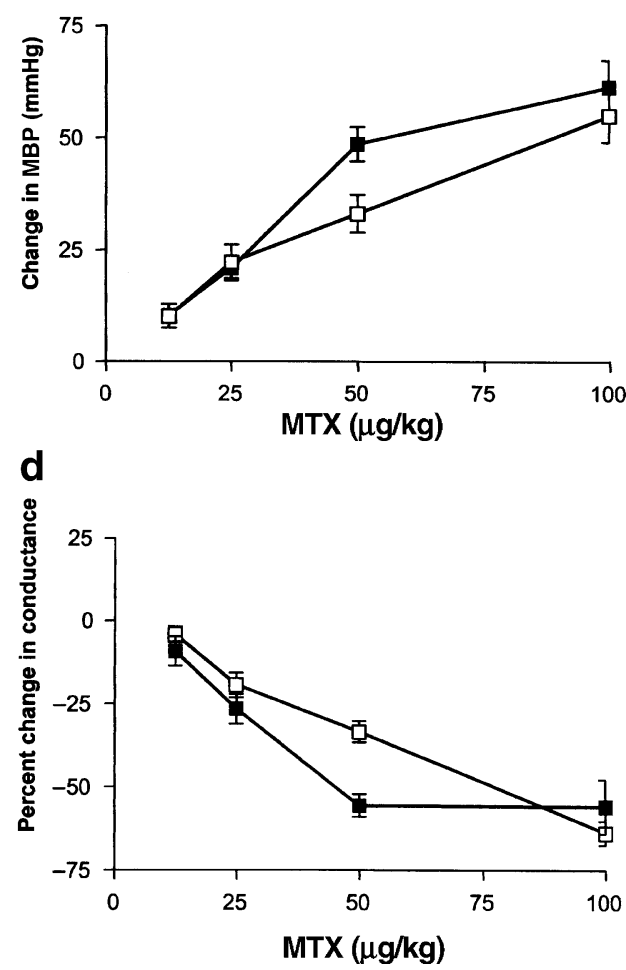


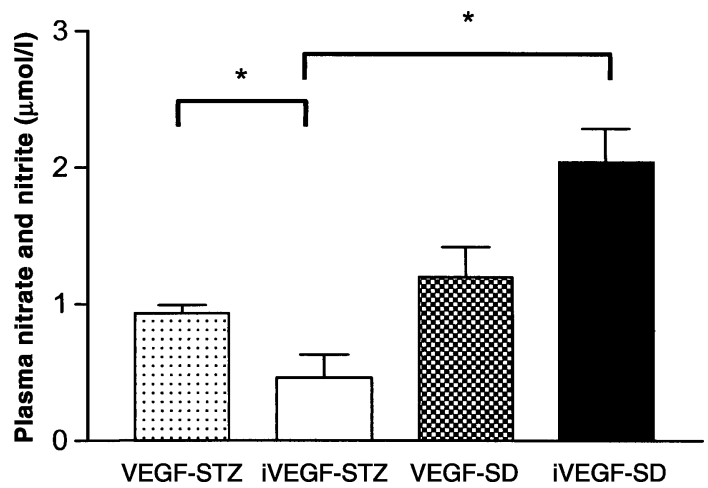

Fig. 4 Effect of chronic treatment with VEGF on levels of plasma nitrate and nitrite in STZ and non-diabetic Sprague-Dawley (SD) rats $\left(n=7\right.$ in each group). Values are mean \pm SEM. ${ }^{*} p<0.05$

counterintuitive. This may be explained by factors that inactivate NO, such as the superoxide anion [17]. By preventing this inactivation, VEGF could maintain NO availability and preserve acetylcholine-evoked responses. Indeed, superoxide anion levels were elevated in STZ diabetic rats, and treatment with VEGF reduced the superoxide anion level to that observed in control SD rats. Thus, our findings on plasma nitrate and nitrite concentrations and on superoxide anion generation are consistent with the interpretation that the availability of $\mathrm{NO}$ is inversely correlated with the magnitude of superoxide anion generation. VEGF has been reported to upregulate the expression of superoxide dismutase, a major superoxide scavenger, in endothelial cells [7]. Moreover, reductions in superoxide dismutase have been reported to inhibit endothelium-dependant relaxation through the inactivation of NO [4]. The pivotal role of the superoxide anion in mitigating responses to acetylcholine is not surprising as reactive oxygen species have long been thought to play a key role in diabetic vascular dysfunction $[18,19]$. Vitamin $\mathrm{C}$, vitamin $\mathrm{E}$ and superoxide dismutase have all been used successfully in restoring agonist-evoked endothelial responses [20-22].

Another finding of the present study is the effect of VEGF treatment on baseline haemodynamics in STZ rats.

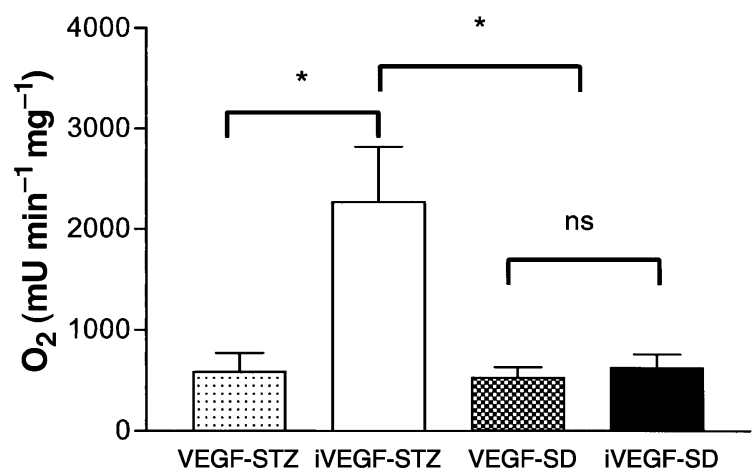

Fig. 5 Effect of chronic VEGF treatment on aortic superoxide anion levels in STZ ( $n=4$ for VEGF-STZ, $n=6$ for iVEGF-STZ) and nondiabetic Sprague-Dawley (SD) rats $(n=3$ for VEGF-SD, $n=4$ for iVEGF-SD). Values (mean \pm SEM) are expressed as $\mathrm{mLL} \mathrm{min}^{-1}$ $\mathrm{mg}^{-1}$ of rat aorta. ${ }^{*} p<0.05$

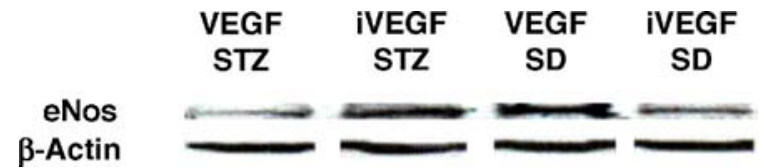

Fig. 6 Effect of chronic treatment with VEGF on expression of eNOS in STZ and non-diabetic Sprague-Dawley (SD) rats. Western blot analyses were performed on sections of aortic arch from STZ and non-diabetic SD rats treated with either VEGF or iVEGF, using a monoclonal antibody to eNOS

The lower baseline arterial pressure observed in STZdiabetic rats compared with control SD rats (Table 1) is consistent with previous reports in the literature [23]. In conscious, chronically instrumented rats, Tatchum-Talom et al. reported a progressive lowering of blood pressure during the 5-week period following induction of the diabetic state with STZ [24]. This is not likely to be an effect of STZ itself as the hypotension is progressive, not becoming significant until the third week of diabetes in conscious animals. The fall in blood pressure could be related to the dilated state of the vasculature. In fact, SMA conductance was much higher in STZ-diabetic rats than in SD rats. Alternatively, the lower blood pressure could be related to hypovolaemia secondary to osmotic diuresis, or to myocardial dysfunction $[25,26]$. Chronic treatment with VEGF was able to preserve baseline MBP in anaesthetised STZ rats, but this was not accompanied by corresponding decreases in SMA conductance. Thus, the preservation of blood pressure must be related to the resistance function of other vascular beds or to the maintenance of cardiac output by preventing osmotic diuresis or myocardial dysfunction $[25,26]$.

Significantly improved agonist-evoked responses to both ET-1 and methoxamine were secondary findings of this study. Several studies have reported increased levels of and attenuated responses to ET-1 in experimental and clinical type 1 and 2 diabetes [10, 27-29]. If indeed VEGF is able to maintain the availability of NO in STZ rats, this might result in a decrease in the release of ET-1 [30]. Decreasing release may also have significant pathophysiological consequences, as this vasoconstrictor peptide is also able to increase vascular superoxide levels [31]. Beyond simply preserving vascular responses to this potent vasoconstrictor, reducing the synthesis of ET-1 with VEGF may help to mitigate another source of vascular superoxide in diabetes.

The mechanism behind the ability of VEGF to preserve methoxamine responses is less clear. Both attenuated and exaggerated responses to $\alpha-1$ agonists have been reported $[11,32]$. The ability of VEGF to activate protein kinase C, through both flt-1 and flk-1 receptors, has been reported [33]. Such an action of VEGF could conceivably contribute to the improved responses to methoxamine, but further discussion of this plausible mechanism is speculative at this point.

The angiogenic growth factor VEGF plays a key and dichotomous role in diabetic vascular complications [34, 35]. Hence the central pathophysiological feature of proliferative diabetic retinopathy is VEGF-stimulated an- 
giogenesis, and experimental therapies target inhibition of VEGF [36]. Conversely, the critical limb ischaemia that is one of the defining features of diabetes is due to impaired peripheral angiogenic responses to ischaemia, and has been successfully treated using VEGF [37]. This dichotomy is one of the true mysteries associated with this disease, but when it is fully understood it may provide investigators with a more effective way to manage the devastating vasculopathy characteristic of diabetes. The data reported in this article demonstrate the ability of VEGF to preserve both depressor and pressor agonist responses. The results emphasise a potential role for this growth factor in the effort to preserve the functional integrity of the vasculature even when the hyperglycaemia associated with the diabetic state is uncontrolled. A possible therapeutic role for VEGF in the management of diabetic vascular complications merits further investigation.

Acknowledgements The authors would like to acknowledge the expert technical assistance of D. Brown, B. Duchek and B. Wilcox. VEGF was a generous donation provided by Genentech, South San Francisco, CA, USA. Funding support was provided by the Canadian Institutes for Health Research (CIHR) and the Heart and Stroke Foundation of Saskatchewan.

\section{References}

1. King GL, Sheetz MJ (2002) Molecular understanding of hyperglycemia's adverse effects for diabetic complications. JAMA 288:2579-2588

2. Calles-Escandon J, Cipolla M (2001) Diabetes and endothelial dysfunction: a clinical perspective. Endocr Rev 22:36-52

3. Kamata K, Miyata N, Kasuya Y (1989) Impairment of endothelium-dependent relaxation and changes in levels of cyclic GMP in aorta from streptozotocin-induced diabetic rats. Br J Pharmacol 97:614-618

4. Lynch SM, Frei B, Morrow JD et al (1997) Vascular superoxide dismutase deficiency impairs endothelial vasodilator function through direct inactivation of nitric oxide and increased lipid peroxidation. Arterioscler Thromb Vasc Biol 17:2975-2981

5. Diabetes Control and Complications Trial Research Group (1993) The effects of intensive diabetes treatment on the development and progression of long-term complications in insulin-dependent diabetes mellitus: the diabetes control and complications trial. N Engl J Med 329:977-986

6. Genuth S (1996) Exogenous insulin administration and cardiovascular risk in non-insulin-dependent and insulin-dependent diabetes mellitus. Ann Intern Med 124:104-109

7. Fukuo K, Yang J, Yasuda O et al (2002) Nifedipine indirectly upregulates superoxide dismutase expression in endothelial cells via vascular smooth muscle cell-dependent pathways. Circulation 106:356-361

8. Johnstone MD, Creager SJ, Scales KM et al (1993) Impaired endothelium-dependent vasodilation in patients with insulindependent diabetes mellitus. Circulation 88:2510-2516

9. Fulton DJ, Hodgson WC, Sikorski BW, King RG (1991) Attenuated responses to endothelin-1, $\mathrm{KCl}$ and $\mathrm{CaCl} 2$, but not noradrenaline, of aortae from rats with streptozotocin-induced diabetes mellitus. Br J Pharmacol 104:928-932

10. Cameron NE, Cotter MA (1992) Impaired contraction and relaxation in aorta from streptozotocin-diabetic rats: role of polyol pathway. Diabetologia 35:1011-1019
11. Misurski DA, Hopfner RL, Gopalakrishnan V (2001) Attenuated agonist evoked vasoconstrictor responses in the perfused mesenteric vascular bed of streptozotocin diabetic rats. Exp Biol Med (Maywood) 226:940-946

12. Meurice T, Bauters C, Vallet B et al (1997) bFGF restores endothelium-dependent responses of hypercholesterolemic rabbit thoracic aorta. Am J Physiol 272:H613-H617

13. Meurice T, Bauters C, Vallet B et al (1997) Basic fibroblast growth factor restores endothelium-dependent responses after balloon injury of rabbit arteries. Circulation 93:18-22

14. Shen BQ, Lee DY, Ziocheck TF (1999) Vascular endothelial growth factor governs endothelial nitric-oxide synthase expression via a KDR/Flk-1 receptor and a protein kinase $\mathrm{C}$ signaling pathway. J Biol Chem 274:33057-33063

15. Bouloumie A, Schini-Kerth VB, Busse R (1999) Vascular endothelial growth factor up-regulates nitric oxide synthase expression in endothelial cells. Cardiovasc Res 41:773-780

16. Hink U, Li H, Mollnau H et al (2001) Mechanisms underlying endothelial dysfunction in diabetes mellitus. Circ Res 88: E14-E22

17. Gryglewski RJ, Palmer RM, Moncada S (1986) Superoxide anion is involved in the breakdown of endothelium-derived vascular relaxing factor. Nature 320:454-456

18. Guzik TJ, Mussa S, Gastaldi D et al (2002) Mechanisms of increased vascular superoxide production in human diabetes mellitus: role of $\mathrm{NAD}(\mathrm{P}) \mathrm{H}$ oxidase and endothelial nitric oxide synthase. Circulation 105:1656-1662

19. Giugliano D, Ceriello A, Paolisso G (1996) Oxidative stress and diabetic vascular complications. Diabetes Care 19:257-267

20. Timimi FK, Ting HH, Haley EA, Roddy MA, Ganz P, Creager MA (1998) Vitamin C improves endothelium-dependent vasodilation in patients with insulin-dependent diabetes mellitus. J Am Coll Cardiol 31:552-557

21. Skyrme-Jones RA, O'Brien RC, Berry KL, Meredith IT (2000) Vitamin $\mathrm{E}$ supplementation improves endothelial function in type I diabetes mellitus: a randomized, placebo-controlled study. J Am Coll Cardiol 36:94-102

22. Zanetti M, Sato J, Katusic ZS, O'Brien T (2001) Gene transfer of superoxide dismutase isoforms reverses endothelial dysfunction in diabetic rabbit aorta. Am J Physiol Heart Circ Physiol 280:H2516-H2523

23. Rebolledo A, Ayala-Paredes F, Milesi V, Grassi AO, Rinaldi GJ (2001) Short-term streptozotocin-induced diabetes induces blood pressure decrease associated with reduced aortic (45)Ca $(2+)$ uptake and selective depression of the sustained noradrenergic contraction. Diabetes Metab 27:40-48

24. Tatchum-Talom R, Gopalakrishnan V, McNeill JR (2000) Radiotelemetric monitoring of blood pressure and mesenteric arterial bed responsiveness in rats with streptozotocin-induced diabetes. Can J Physiol Pharmacol 78:721-728

25. Schaan BD, Dall'Ago P, Maeda CY et al (2004) Relationship between cardiovascular dysfunction and hyperglycemia in streptozotocin-induced diabetes in rats. Braz J Med Biol Res 37:1895-1902

26. Hebden RA, Gardiner SM, Bennett T, MacDonald IA (1986) The influence of streptozotocin-induced diabetes mellitus on fluid and electrolyte handling in rats. Clin Sci (Lond) 70: $111-117$

27. Nugent AG, McGurk C, Hayes JR, Johnston GD (1996) Impaired vasoconstriction to endothelin 1 in patients with NIDDM. Diabetes 45:105-107

28. Kawamura M, Ohgawara H, Naruse $M$ et al (1992) Increased plasma endothelin in NIDDM patients with retinopathy. Diabetes Care 15:1396-1397

29. Takahashi K, Ghatei MA, Lam HC, O'Halloran DJ, Bloom SR (1990) Elevated plasma endothelin in patients with diabetes mellitus. Diabetologia 33:306-310

30. Boulanger C, Luscher TF (1990) Release of endothelin from the porcine aorta. Inhibition by endothelium-derived nitric oxide. J Clin Invest 85:587-590 
31. Li L, Fink GD, Watts SW et al (2003) Endothelin-1 increases vascular superoxide via endothelin A-NADPH oxidase pathway in low-renin hypertension. Circulation 107:1053-1058

32. Weber LP, Macleod KM (1997) Influence of streptozotocin diabetes on the alpha-1 adrenoceptor and associated $\mathrm{G}$ proteins in rat arteries. J Pharmacol Exp Ther 283:1469-1478

33. Xia P, Aiello LP, Ishii H et al (1996) Characterization of vascular endothelial growth factor's effect on the activation of protein kinase $\mathrm{C}$, its isoforms, and endothelial cell growth. J Clin Invest 98:2018-2026

34. Duh E, Aiello LP (1999) Vascular endothelial growth factor and diabetes: the agonist versus antagonist paradox. Diabetes 4810:1899-1906
35. Aiello LP, Wong JS (2000) Role of vascular endothelial growth factor in diabetic vascular complications. Kidney Int [Suppl] 77:S113-S119

36. Witmer AN, Vrensen GF, Van Noorden CJ, Schlingemann RO (2003) Vascular endothelial growth factors and angiogenesis in eye disease. Prog Retin Eye Res 22:1-29

37. Isner JM, Pieczek A, Schainfeld R et al (1996) Clinical evidence of angiogenesis after arterial gene transfer of phVEGF165 in patient with ischaemic limb. Lancet 348: 370-374 